\title{
Suicide Risk across Latent Class Subgroups: A Test of the Generalizability of the Interpersonal Psychological Theory of Suicide
}

\author{
Jennifer S. Ma, BPsy (Hons) iD, Philip J. Batterham, MPH, PhD iD, Alison L. \\ Calear, PhD, and Jin Han, MSc
}

It remains unclear whether the Interpersonal Psychological Theory of Suicide (IPTS; Joiner, 2005) is generalizable to the population or holds more explanatory power for certain subgroups compared to others. The aim of this study was to (1) identify subgroups of individuals who endorsed suicide ideation in the past month based on a range of mental health and demographic variables, (2) compare levels of the IPTS constructs within these subgroups, and (3) test the IPTS predictions for suicide ideation and suicide attempt for each group. Latent class, negative binomial, linear, and logistic regression analyses were conducted on population-based data obtained from 1,321 adults recruited from Facebook. Among participants reporting suicide ideation, four distinct patterns of risk factors emerged based on age and severity of mental health symptoms. Groups with highly elevated mental health symptoms reported the highest levels of thwarted belongingness and perceived burdensomeness. Tests of the IPTS interactions provided partial support for the theory, primarily in young adults with elevated mental health symptoms. Lack of support found for the IPTS predictions across the subgroups and full sample in this study raise some questions around the broad applicability of the theory.

Worldwide, it is estimated that over 800,000 people die by suicide each year (World Health Organization, 2014). Although preventable, suicide remains a major public health concern and there persists a need for the better identification of suicide risk and protective factors, and more accurate prediction of its development (Franklin et al., 2016). The Interpersonal Psychological Theory of Suicide (IPTS; Joiner, 2005; Van Orden et al., 2010) was developed to provide testable predictions of who will develop a desire for suicide (i.e., ideation) and who will go on to attempt. The theory proposes that suicidal desire is caused by the simultaneous presence of two proximal, causal risk factors: thwarted belongingness (TB), the experience that one is alienated from friends, family, or other valued social circles; and perceived burdensomeness (PB), the view that one's existence is a burden on friends, family members, and/or society (Van Orden et al., 2010). Both are dynamic cognitive-affective states influenced by inter- and intrapersonal factors, such as family conflict, living alone, and having few social supports
Jennifer S. Ma, Philip J. Batterham, and Alison L. Calear, Center for Mental Health Research, Research School of Population Health, The Australian National University, Canberra, ACT, Australia.
Address correspondence to Jennifer Ma, Centre for Mental Health Research, The Australian National University, 63 Eggleston Road, Canberra, ACT 2601, Australia; E-mail: jennifer. ma@anu.edu.au 
for TB, and feelings of low self-esteem for PB (Van Orden et al., 2010).

Suicidal behavior is said to only develop through the presence of suicidal desire and an additional third construct: capability for suicide (CS), which refers to one's ability to overcome the inherent drive for self-preservation and engage in lethal self-injury through repeated exposure and habituation to physically painful and/or fear-inducing experiences (Joiner, 2005). Capability for suicide is said to have an underlying genetic component, where risk factors such as family history of suicide, prior suicide attempt, exposure to combat, and childhood maltreatment are said to contribute (Smith et al., 2012; Van Orden et al., 2010). According to the theory, individuals who exhibit high levels of all three interpersonal risk factors are most at risk for lethal suicidal behavior, as they possess both the desire for suicide as well as the capability to enact it.

Research conducted on the IPTS has provided support for the main effects of the interpersonal risk factors across various populations, including community (Christensen, Batterham, Mackinnon, Donker, \& Soubelet, 2014), undergraduate (Kleiman, Law, \& Anestis, 2014), clinical (Czyz, Berona, \& King, 2015), older adult (Cukrowicz, Jahn, Graham, Poindexter, \& Williams, 2013), indigeneous and ethnic (O'Keefe et al., 2014; Wong, Koo, Tran, Chiu, \& Mok, 2011), lesbian-gay-bisexual-transgender (Kim \& Yang, 2015), detainee (Smith et al., 2015), and military (Bryan, Clemans, \& Hernandez, 2012) populations. However, support for the theory's interaction effects has been scarce in comparison, and it remains unclear as to whether the theory holds more explanatory power for certain subgroups compared to others (Ma, Batterham, Calear, \& Han, 2016). This is an important area to investigate as the IPTS assumes equivalent predictive power across individuals.

One way to explore this is to identify subgroups of individuals with suicide ideation is through the use of latent class analysis (LCA; Lazarsfeld \& Henry, 1968), a statistical modeling technique that allows heterogeneous individuals from a population to be grouped into smaller subgroups based on similar characteristics or patterns of behaviors. Suicide studies using LCA have identified subclasses of individuals with suicide ideation or attempt who display different symptom patterns and risk trajectories over time (Logan, Hall, \& Karch, 2011; Rueter, Holm, McGeorge, \& Conger, 2008). Such research highlights the feasibility of classifying individuals based on suicide risk. However, there has been little research using LCA to examine the IPTS interpersonal risk factors, which would enable exploration of potential patterns of co-occurrence between the interpersonal and other known suicide risk factors to aid risk identification and the development of targeted prevention strategies. Of the few studies conducted, results have supported the IPTS, with levels of the interpersonal risk factors found to be higher in suicidal versus nonsuicidal groups (Dhingra, Boduszek, \& Klonsky, 2016; Wong \& Maffini, 2011). However, a limitation of these studies is that they were based on student samples, where typologies may not generalize to community or clinical populations, and middle-aged and older adults are underrepresented. Additionally, none of these studies focused on assessing the full predictions of the IPTS across the subgroups. This is important for identifying the strength of the theory's critical interaction predictions and generalizability across subgroups.

Thus, the aim of the present study was to (1) identify subgroups of individuals who endorsed suicide ideation in the past month from an online community sample based on a range of mental health and demographic variables, (2) compare levels of the IPTS constructs (TB, PB, CS) in these subgroups, and (3) test the theory's predictions for severity of suicide ideation and presence of suicide attempt within each group. For the comparison of IPTS constructs, it was hypothesized that the highest levels of $\mathrm{TB}$ and $\mathrm{PB}$ would be found in groups reporting high levels of mental health symptoms, a strong risk factor for 
suicide. In line with IPTS predictions, it was also hypothesized that across all identified latent classes, the two-way interaction between TB and $\mathrm{PB}$ would be significantly associated with presence of suicide ideation in the past month. Likewise, it was hypothesized that the three-way interaction between TB, PB, and CS would be significantly associated with presence of suicide attempt in the past year.

\section{METHODS}

\section{Participants and Procedure}

Australian adults $(N=1,321 ; \quad 58 \%$ female) aged 18 years and over were recruited from Facebook using targeted paid advertisements linked to an online survey for a study of mental health and suicide ideation outcomes in a population-based sample (Batterham, 2014; Batterham, Calear, \& Christensen, 2013). Sample characteristics are reported in Table 1 . The study involved a 30-minute online survey that assessed psychological distress, depression, anxiety disorders, alcohol use, sleep problems, suicide ideation, suicide literacy, suicide stigma, exposure to suicide, interpersonal risk factors for suicide, and a range of sociodemographic characteristics. Written information about the study aims was provided to participants prior to commencing the survey, with informed consent and a list of mental health resources provided online. No incentive was provided. There was no missing data, and diagnostic analyses revealed no systematic outliers. The study received ethics approval from the Science and Medical Delegated Ethics Review Committee at the Australian National University (protocol number 2012/ 310).

\section{Measures}

Sociodemographic Variables. Gender (reference males), age (18-24, 25-29, 30-39, $40-49,50-59,60$ and over), level of education (up to high school, associate/trade degree or diploma, bachelor's degree, postgraduate degree), employment status (fulltime, part-time, unemployed/seeking work, retired, or not in the workforce), and marital status (married or de facto, single/never married, separated or divorced, widowed) were measured.

Suicide Outcome Measures. Suicide ideation was measured using the Suicidal Ideation Attributes Scale (SIDAS; Van Spijker et al., 2014), which consists of five items that measure the frequency, controllability, and distress of suicidal thoughts, closeness of making an attempt, and impact on daily functioning experienced in the past month on a scale from 0 (never) to 10 (always). Higher scores indicate more severe suicidal thoughts (range 0-50). The SIDAS has strong internal consistency and convergent validity with other measures of suicide and psychological distress (Van Spijker et al., 2014) and demonstrated good internal consistency in this sample $(\alpha=.85)$.

Suicide attempt was measured with the sixth item from the Columbia-Suicide Severity Rating Scale (C-SSRS; Posner et al., 2011) that assesses whether the individual has done anything, started to do anything, or prepared to do anything to end their life in the past year on a yes/no scale. The C-SSRS has good convergent and divergent validity with other multiinformant suicide ideation and behavior scales, and high sensitivity and specificity for suicidal behavior classifications (Posner et al., 2011). Mental Health Measures for Latent Class Analysis. Psychological distress was assessed using the K6 (Kessler et al., 2002), consisting of six items that measure the frequency of negative emotional states experienced over the past 4 weeks on a scale from 0 (none of the time) to 4 (all of the time). Higher scores indicate greater levels of distress (range 0-24). The K6 has been validated in a number of countries (Fassaert et al., 2009; Patel et al., 2008) and has good concordance with independent clinical ratings of serious mental illness. In this sample, the K6 had good internal consistency $(\alpha=.89)$. 


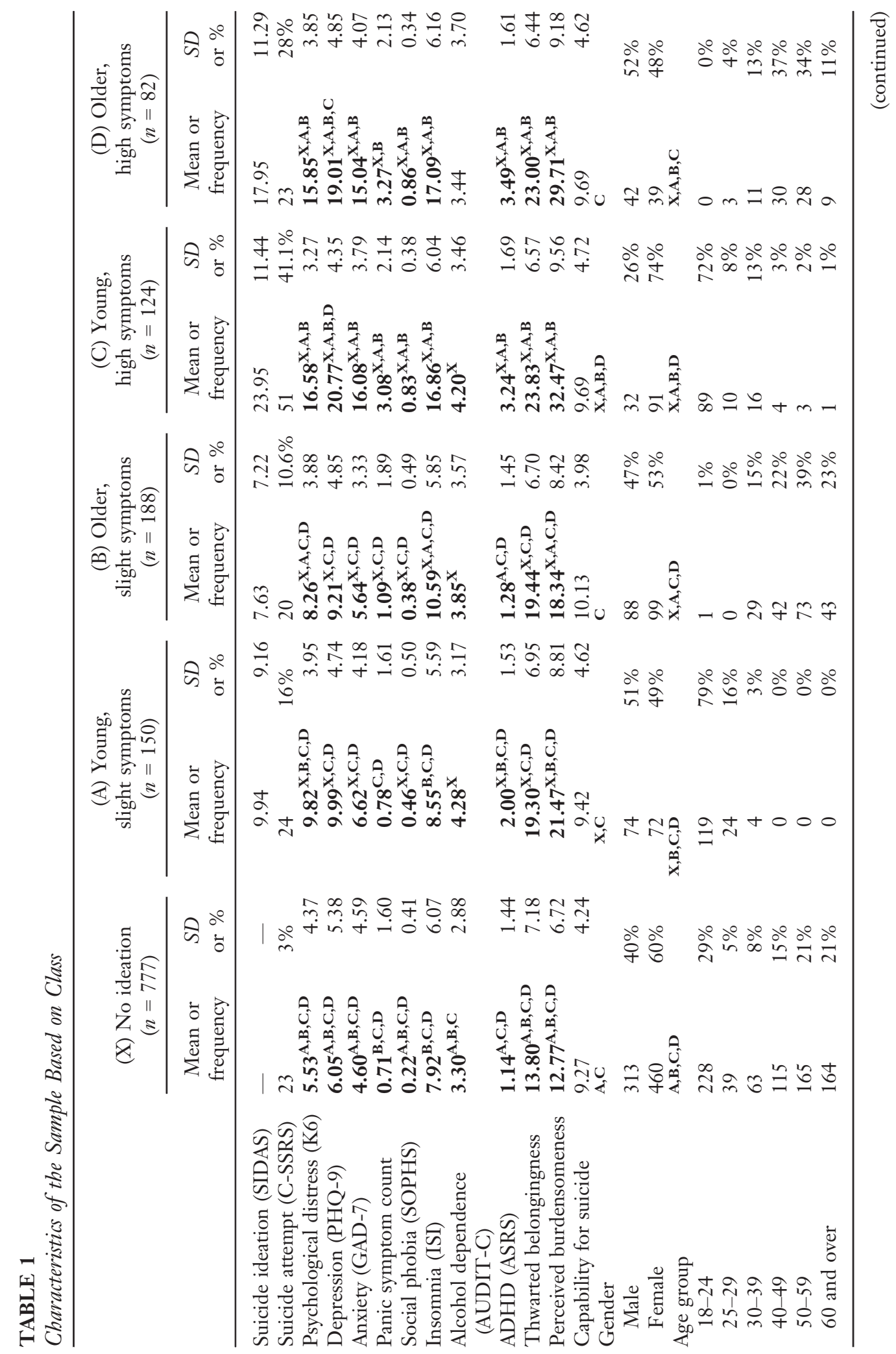




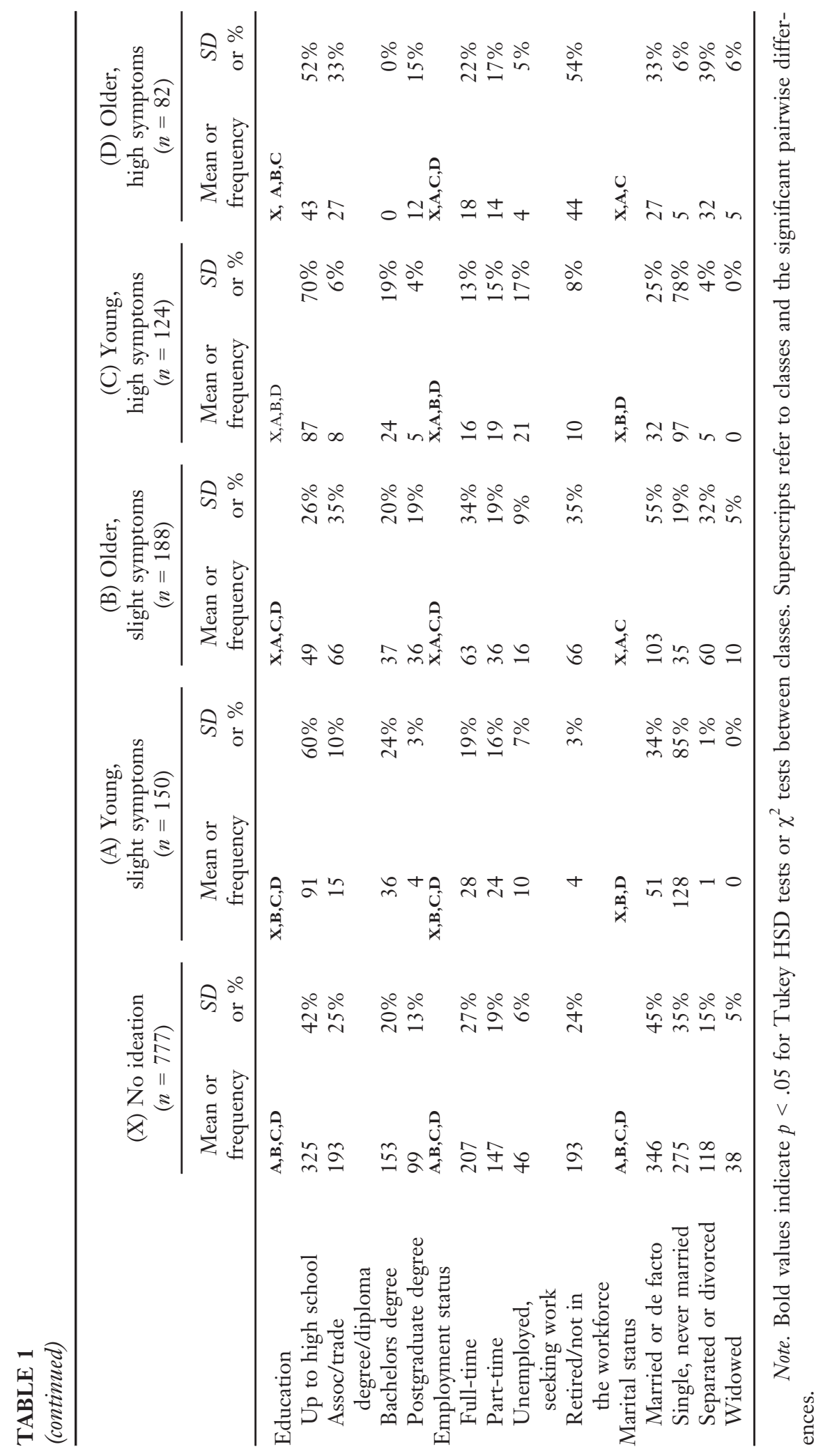


Depression was assessed using the Patient Health Questionnaire (PHQ; Spitzer, Kroenke, \& Williams, 1999) consisting of nine items that measure how often an individual has been bothered by symptoms of depression over the past 2 weeks on a scale from 0 (not at all) to 3 (nearly every day). Higher scores indicate more severe depression symptoms (range 0-27). The PHQ-9 detects major depression with 88\% sensitivity and specificity (Kroenke, Spitzer, \& Williams, 2001) and demonstrated excellent internal consistency $(\alpha=.91)$ in this sample. In this study, the ninth item related to suicide ideation was omitted from the total score to avoid confounding with the outcome.

Anxiety was measured using the Generalized Anxiety Disorder Scale (GAD-7; Spitzer, Kroenke, Williams, \& Löwe, 2006) consisting of seven items that measure how often an individual has been bothered by symptoms of anxiety over the past 2 weeks on a scale from 0 (not at all sure) to 3 (nearly every day). Higher scores indicate more severe anxiety symptoms (range $0-21$ ). The GAD-7 detects generalized anxiety disorder with $89 \%$ sensitivity and $82 \%$ specificity (Spitzer et al., 2006) and demonstrated excellent internal consistency $(\alpha=.92)$ in this sample.

Panic symptom count was measured using the PHQ-Panic (Spitzer et al., 1999) consisting of 15 items that measure the presence and characteristics of anxiety attacks experienced over the past 4 weeks on a yes/no scale. The PHQ-Panic has been validated in high-risk primary care and outpatient populations (Löwe et al., 2003; Wittkampf, Baas, van Weert, Lucassen, \& Schene, 2011). In this sample, the PHQPanic had excellent internal consistency $(\mathrm{KR}-20=.95)$.

Social phobia was measured using the Social Phobia Screener (SOPHS; Batterham, Mackinnon, \& Christensen, 2016) consisting of five items that measure the presence and extent of fear and embarrassment experienced over the past month on a scale from 1 (not at all) to 5 (extremely).
Higher scores indicate greater severity (range 5-25). The SOPHS has been validated in a community-based sample of young Australians aged 18-30 $(n=12,292)$ (Batterham et al., 2016). In this sample, the SOPHS had excellent internal consistency $(\alpha=.91)$.

Insomnia was measured using the Insomnia Severity Index (ISI; Bastien, Vallières, \& Morin, 2001) consisting of seven items that target the subjective symptoms and consequences of insomnia and sleep concerns experienced over the last 2 weeks on a scale from 0 (none) to 4 (very severe). Higher scores indicate more acute symptoms of insomnia (range 0-28). The ISI has concurrent validity with clinician ratings and subjective and objective sleep measures (Bastien et al., 2001), and had good internal consistency $(\alpha=.86)$ in this sample.

Alcohol dependence was measured using the AUDIT-C (Bush, Kivlahan, McDonell, Fihn, \& Bradley, 1998) consisting of three items that measure the frequency of alcohol consumption across a month, week, and typical day on a fivepoint scale. Higher scores indicate that the respondent's drinking is affecting their safety (range 0-12). The AUDIT-C has a sensitivity of $86 \%$ and specificity of $72 \%$ in detecting individuals with heavy drinking or dependence (Bush et al., 1998). In the current sample, the AUDIT-C had acceptable internal consistency $(\alpha=.73)$.

Attention deficit hyperactivity disorder (ADHD) was measured using the ADHD Self-Report Scale (ASRS; Kessler et al., 2005) consisting of six items that measure how often an individual has had difficulties with organization and overactivity over a 6 month period on a scale from 0 (never) to 4 (very often). Higher scores indicate greater severity of ADHD symptoms (range 0-24). The ASRS screener has adequate sensitivity $(68.7 \%)$ and excellent specificity (99.5\%; Kessler et al., 2005), and demonstrated good internal consistency $(\alpha=.80)$ in the current sample.

Interpersonal Risk Factors. $\mathrm{TB}$ and $\mathrm{PB}$ were measured using the Interpersonal 
Needs Questionnaire (INQ-12; Van Orden, Cukrowicz, Witte, \& Joiner, 2012; Van Orden, Witte, Gordon, Bender, \& Joiner, 2008) consisting of five items that assess TB and seven that assess PB on a scale from 1 (not at all true for me) to 7 (very true for me). Higher ratings indicate greater TB (range 5-35) and PB (range 7-49). The INQ-12 has been validated in community and undergraduate samples (Batterham, Calear, \& Spijker, 2015; Freedenthal, Lamis, Osman, Kahlo, \& Gutierrez, 2014). In this sample, the INQ-12 had excellent internal consistency $(\alpha=.91)$, the TB subscale had good internal consistency $(\alpha=.85$ ), and the PB subscale had excellent internal consistency $(\alpha=.91)$.

CS was measured using the Acquired Capability for Suicide Scale (ACSS; Van Orden et al., 2008) consisting of five items that measure fearlessness about engaging in potentially lethal self-harmful behaviors on a scale from 1 (not at all like me) to 5 (very much like me). Higher scores indicate greater capability for suicide (range 5-25). This short form of the ACSS has been validated in a community sample (Batterham et al., 2015). In this sample, the ACSS had questionable internal consistency $(\alpha=.63)$. Therefore, analyses were re-estimated using the first three items of the ACSS that better fit a unidimensional construct $(\alpha=.72)$. Findings from the re-estimated models were largely consistent with the analyses presented.

\section{Analysis}

Latent class analysis (LCA; Lazarsfeld \& Henry, 1968) was conducted with Mplus version 6.12 (Muthén \& Muthén, 19982010) among participants who reported suicide ideation in the past month $(n=544)$. LCA is a form of mixture modeling that aims to categorize people into classes using observed dependent variables and identify items that best distinguish between the classes (Nylund, Asparouhov, \& Muthén, 2007). LCA estimation procedures assign respondents to groups based on probability estimates (i.e., the combined probability that a proportion of the population would fall into a given suicide ideation class and that a particular response to the self-report measures would occur) from which statistical fit indices can be used to evaluate competing models.

In the study, LCA was used to identify class membership based on a number of mental health and demographic variables including psychological distress, depression, generalized anxiety, panic symptoms, social anxiety, insomnia, alcohol dependence, ADHD symptoms, gender, age, level of education, employment status, and martial status. First, a one-class model was specified and run, repeating analyses until the addition of classes was found to have no significant improvement on model fit, based on the bootstrapped likelihood ratio test (BLRT; McLachlan \& Peel, 2000). After the best fitting model was identified, names were generated to broadly describe the characterization of the overall response patterns.

Following the LCA, one-way ANOVAs and post hoc follow-up testing (i.e., Tukey) were conducted to compare levels of the interpersonal risk factors (i.e., TB, PB, CS) across each class. Differences in mental health variables across the classes were assessed by Tukey HSD tests and demographic differences by $\chi^{2}$ tests. Refusals to answer questions on age $(n=8$ or $0.6 \%)$, education ( $n=11$ or $0.8 \%)$, employment $(n=17$ or $1.3 \%)$, and marital status ( $n=26$ or $2.0 \%)$ were treated as missing values. Linear regression models were then used to test the IPTS predictions for severity of suicide ideation reported over the previous month, and logistic regression models to test the IPTS predictions for suicide attempt reported over the previous year across the classes. As the suicide ideation outcome displayed over dispersion in the full sample (LR $\chi^{2}=1874.17, d f=1$, $p<.01)$ and classes A (LR $\chi^{2}=520.50$, $d f=1, p<.01)$ and B (LR $\chi^{2}=468.73$, $d f=1, p<.01)$, negative binomial regression models were used. To account for the presence of excess zeros in the full sample, 
zero inflated negative binomial regression was employed. The suicide ideation model included the main effects of $\mathrm{TB}, \mathrm{PB}$, and their two-way interaction, as the model indicates that high levels of both constructs be present for suicidal desire to develop. Similarly, the suicide attempt model included the main effects of TB, PB, CS, and their twoand three-way interactions. The IPTS and suicide ideation variables were standardized to have a mean of 0 and $S D$ of 1 in the linear and logistic regression models to aid interpretation. Descriptive analysis, multiple, and logistic regressions were conducted using SPSS v23 (IBM Corp, New York, 2011). Negative binomial regressions were conducted using STATA v14 (StataCorp, 2015).

\section{RESULTS}

\section{Class Assignment Based on Mental Health and Demographic Variables}

Analysis of the mental health and demographic items from participants who reported suicide ideation in the past month $(n=544)$ indicated that the 4-class model was the best fitting solution as compared to the 1-, 2-, 3-, and 5-class models. Fit indices showed that the parametric BLRT of 3 versus 4 classes was significant $(p<.001)$, indicating better fit for the 4-class model, while the 5-class model did not have significantly better fit than the 4-class model. The $-2 \log$ likelihood was also significant, $\chi^{2}(d f=30$, $n=544)=258.8, p<.001$. None of the classes contained less than $5 \%$ of the sample (the smallest class contained 15\%), and the entropy value of .89 was greater than the recommended .80 .

Membership for the 4-class solution was as follows: Class A had $150(11 \%$ of full sample) members and consisted primarily of individuals 18-29 years old, who were predominantly single, had completed high school, were currently a student, and had slightly elevated mental health scores (young group with slightly elevated symptoms);
Class B had 188 (14\%) members and consisted primarily of individuals aged 30 years and older who were predominantly either single, separated/divorced or widowed, and had slightly elevated mental health scores (older group with slightly elevated symptoms); Class C had 124 (9\%) members and consisted primarily of individuals 18-29 years old who were predominantly female, single, had completed high school, and scored highly across all mental health symptom measures (young group with highly elevated symptoms); and lastly, Class D had 82 (6\%) members and consisted primarily of individuals 30 years and older who were predominantly either married/de facto or separated/divorced, had completed some of high school, were more likely to be retired or not in the workforce, and scored highly across all mental health symptoms (older group with highly elevated symptoms).

All classes differed from Class $\mathrm{X}$ (no suicide ideation, $58 \%$ full sample) on most mental health measures, except for comparisons between Class A on panic symptom count and insomnia, Class B on ADHD, and Class D with symptoms of alcohol dependence. Additionally, all classes significantly differed from Class $\mathrm{X}$ on age, education, marital status, and employment status, which may have been attributable to the age differences.

\section{Class Differences in the Interpersonal Risk Factors}

Three one-way between-groups ANOVA were conducted to explore the impact of class on levels of TB, PB, and CS. $\mathrm{TB}, \mathrm{PB}$, and $\mathrm{CS}$ were the dependent variables, and latent class was the independent variable. For the TB ANOVA, there was a statistically significant difference in TB scores for the five classes: $F(4,1,321)=$ $94.64, p<.001$. The mean difference ranged from 0.13 to 10.03 , with an eta-square effect size of 0.22 . Post hoc comparisons using the Tukey HSD test indicated that the five groups significantly differed from each other on levels of TB, except for the comparisons 
between Classes A and B (the young and older slightly elevated symptom groups), and Classes C and D (the young and older highly elevated symptom groups) (Figure 1). For the PB ANOVA, there was a statistically significant difference at the $p<.001$ level in PB scores for the five classes: $F(4,1,321)=$ $252.66, p<.001$. The mean difference ranged from 2.75 to 19.70 , with an eta-square effect size of 0.43 . Post hoc comparisons using the Tukey HSD test indicated that all five groups significantly differed from each other on levels of $\mathrm{PB}$, except for the comparisons between Classes C and D (the young and older highly elevated symptom groups) (Figure 2). For the CS ANOVA, no statistically significant difference between classes was found: $F(4,1,321)=1.65, p=.15$ (Figure 3).

Testing the Predictions of the IPTS Model on Suicide Ideation and Attempt Outcomes by Class

Zero inflated negative binomial regression models (full sample), negative binomial regression models (Classes $\mathrm{A}$ and $\mathrm{B}$ ), and linear regression models (Classes $\mathrm{C}$ and $\mathrm{D})$ were used to assess associations of the interpersonal risk factors $(\mathrm{TB}, \mathrm{PB}$, and their two-way interaction) with severity of suicide ideation reported in the past month. Across the groups, $25 \%$ in the full sample $(M=13.54, S D=11.53), 13 \%$ in Class A (young, slight symptoms; $M=9.94, S D=$ 9.16), $5 \%$ in Class B (older, slight symptoms; $M=7.63, S D=7.22$ ), $59 \%$ in Class C (young, high symptoms; $M=23.95$, $S D=11.44$ ), and $40 \%$ in Class D (older, high symptoms; $M=17.95, S D=11.29)$ reported a SIDAS severity score in the extreme range $(\geq 21$; Van Spijker et al., 2014). The negative binomial regression model with all three predictors was significant in the full sample (LR $\chi^{2}=171.86$, $d f=3, p<.01$ ), Class A (young, slight symptoms; LR $\left.\chi^{2}=30.07, d f=3, p<.01\right)$ and $\mathrm{B}$ (older, slight symptoms; LR $\left.\chi^{2}=25.80, \quad d f=3, \quad p<.01\right)$. The linear regression models containing all three predictors were also statistically significant, where it explained $30.6 \%$ of the variance in

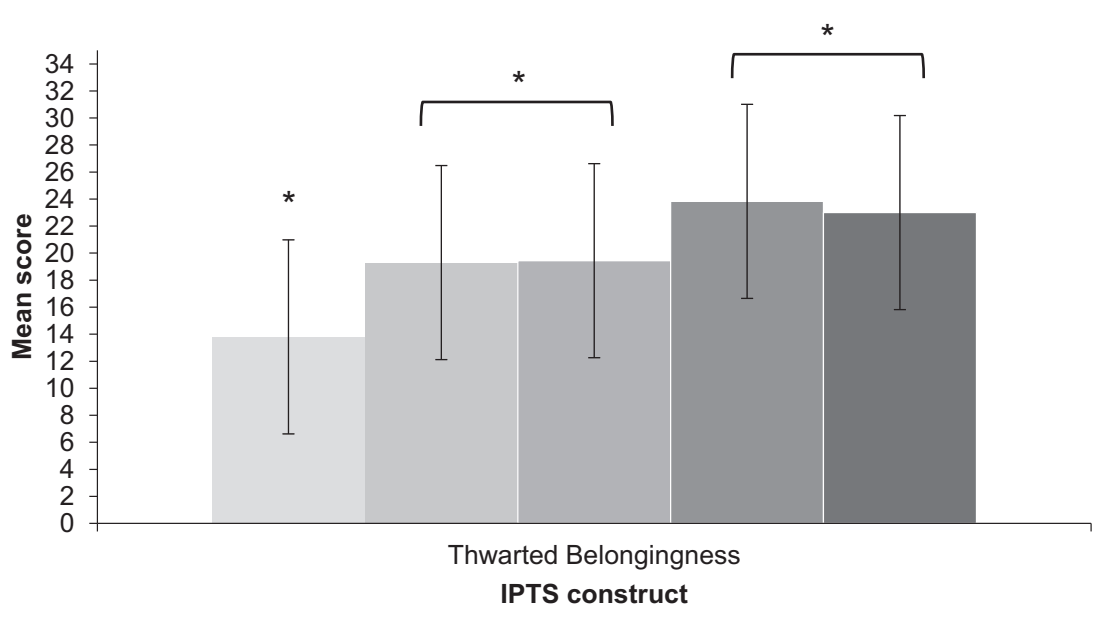

\footnotetext{
$\square(X)$ No suicide ideation $(n=777)$

- (B) Older, S $(n=188)$

- (D) Older, $\mathrm{H}(n=82)$
}

Figure 1. Comparison of levels of thwarted belongingness across classes. IPTS, Interpersonal-Psychological Theory of Suicide, $\mathrm{S}=$ Slightly elevated symptoms, $\mathrm{H}=$ highly elevated symptoms. Error bars represent standard deviation. Significant group differences are indicated by asterisks. 


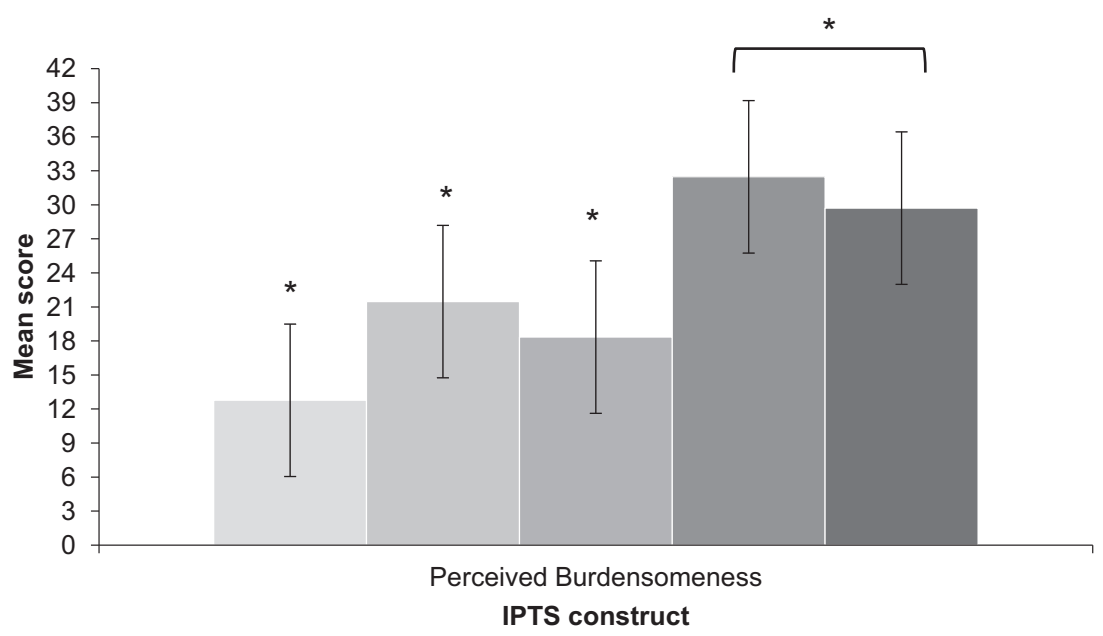

\begin{tabular}{|c|c|}
\hline (X) No suicide ideation $(n=777)$ & (A) Young, S $(n=150)$ \\
\hline (B) Older, S $(n=188)$ & (C) Young, $\mathrm{H}(n=124)$ \\
\hline$=(\mathrm{D})$ Older, $\mathrm{H}(n=82)$ & \\
\hline
\end{tabular}

Figure 2. Comparison of levels of perceived burdensomeness across classes. IPTS, Interpersonal-Psychological Theory of Suicide, $\mathrm{S}=$ Slightly elevated symptoms, $\mathrm{H}=$ highly elevated symptoms. Error bars represent standard deviation. Significant group differences are indicated by asterisks.

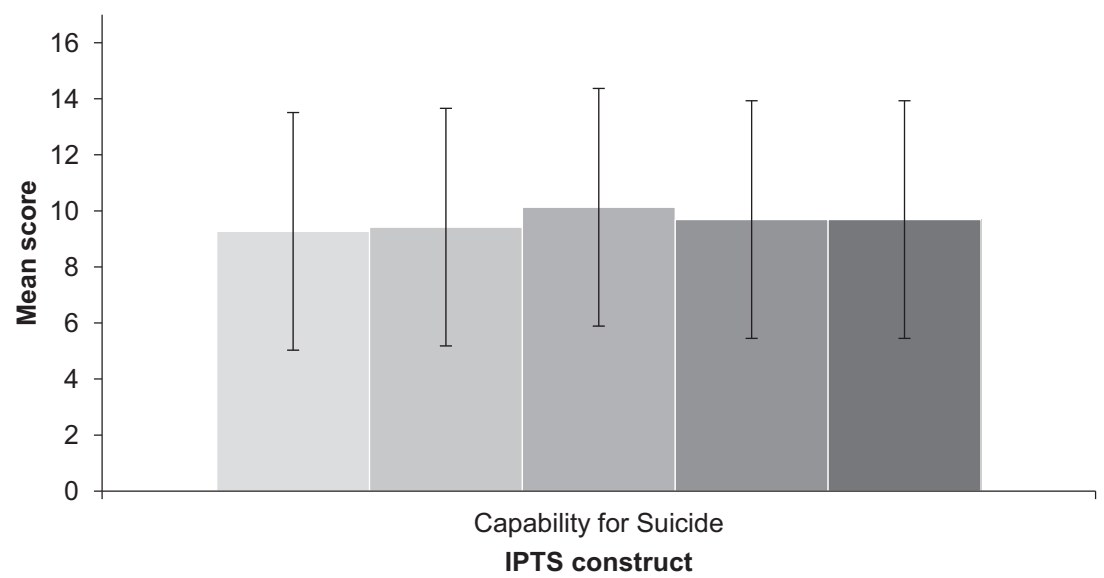

\footnotetext{
$(\mathrm{X})$ No suicide ideation $(n=777) \quad \square(\mathrm{A})$ Young, $\mathrm{S}(n=150)$

-(B) Older, $\mathrm{S}(n=188) \quad \square(\mathrm{C})$ Young, $\mathrm{H}(n=124)$

(D) Older, $\mathrm{H}(n=82)$
}

Figure 3. Comparison of levels of capability for suicide across classes. IPTS, Interpersonal-Psychological Theory of Suicide, S = Slightly elevated symptoms, H = highly elevated symptoms. Error bars represent standard deviation. 
suicide ideation for Class $\mathrm{C}$ (young, high symptoms; $F(3,124)=17.67, p<.001)$, and $16.4 \%$ for Class D (older, high symptoms; $F(3,82)=5.09, p=.003)$. However, despite the significant models across all groups, as shown in Table 2, the two-way interaction of $\mathrm{TB}$ and $\mathrm{PB}$ made a significant contribution only in Class $\mathrm{C}$ (young, high symptoms; $\beta=0.25, p=.01$ ) (Figure 4).

Logistic regression models were used to assess associations of the interpersonal risk factors (TB, PB, CS, and their two-way and three-way interactions) with the likelihood that respondents reported suicide behavior in the past year. Across the groups, $10 \%$ participants in the full sample, $2 \%$ participants in Class X (no suicide ideation), $16 \%$ in Class A (young, slight symptoms), $10 \%$ in Class B (older group, slight symptoms), $41 \%$ in Class C (young, high symptoms), and $28 \%$ in Class D (older, high symptoms) reported suicide behavior in the past year. The full model containing all eight predictors was statistically significant for the full sample, $\chi^{2}(7$, $N=1,321)=194.94, p<.001$; Class $\mathrm{X}$ (no suicide ideation; $\chi^{2}(7, N=777)=40.06$, $p<.01$ ); and Class C (young, high symptoms, $\left.\chi^{2}(7, N=124)=19.84, p<.01\right)$. It was not significant for Class A (young, slight symptoms, $\chi^{2}(7, N=150)=11.44$, $p=.12$ ), Class B (older, slight symptoms, $\left.\chi^{2}(7, N=188)=6.46, p=.48\right)$, or Class D (older, high symptoms, $\chi^{2}(7, \quad N=82)=$ 13.35, $p=.06)$, indicating that the model did not significantly distinguish between respondents who reported and did not report suicide attempt in the past year in these groups. The significant models as a whole explained between 5\% (Cox and Snell R square) to $27.8 \%$ (Nagelkerke R squared) of the variance in suicide attempt, and correctly classified $67.7 \%$ to $97.3 \%$ of cases. The model for the full sample, followed by the model for Class X (no suicide ideation) explained the most variance. As shown in Table 3 and Figure 5, the three-way interaction was only significant in the Class $\mathrm{X}$ model, where respondents who reported experiencing all three

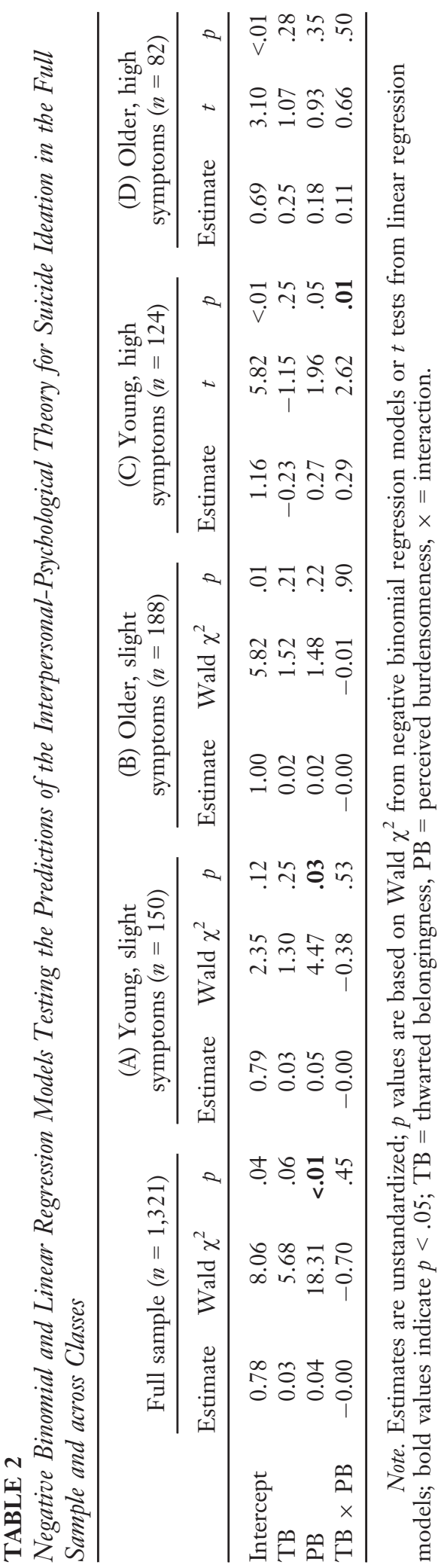




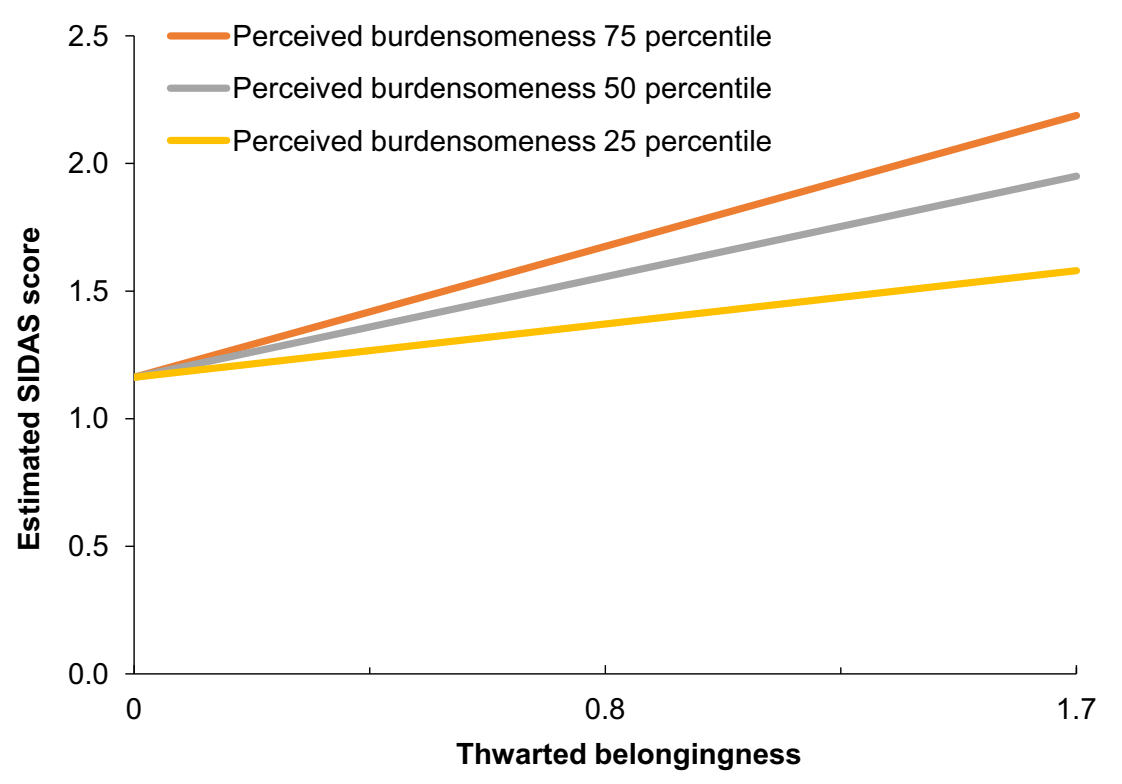

Figure 4. Interaction between thwarted belongingness and perceived burdensomeness (PB) on suicide ideation in Class C (young, high symptoms). TB, PB, and SIDAS scores are standardized. SIDAS, Suicidal Ideation Attributes Scale.

interpersonal risk factors simultaneously were significantly less likely to report having attempted suicide over the past year compared to those who did not, controlling for all other factors in the model. However, on using the three-item version of the ACSS that showed adequate internal consistency, this three-way interaction effect was no longer significant $(p=.08)$.

\section{DISCUSSION}

The value in theories of suicidal behavior is in their ability to identify shared factors that predict whether any particular individual is likely to die by suicide (Gunn \& Lester, 2014). However, limited research has tested whether the IPTS holds more explanatory power for specific subgroups or whether it is broadly applicable. The current study aimed to: (1) identify subgroups of individuals who endorsed suicide ideation in the past month from an online community sample based on a range of mental health and demographic variables, (2) compare levels of the IPTS constructs across these subgroups, and (3) test the IPTS predictions for suicide ideation and suicide attempt for each group. Four distinct classes of individuals differentiated by age and severity of mental health symptoms endorsing suicide ideation in the past month were identified. The majority of the ideation sample were classed into a group that consisted of older individuals $(30-60+)$ with slightly elevated mental health symptom scores, followed by groups consisting of younger individuals (18-29) with slightly elevated mental health symptom scores, young individuals (18-29) with highly elevated mental health symptom scores, and older individuals (30-60+) with highly elevated mental health symptom scores. All classes significantly differed from the no suicide ideation class on most mental health measures in addition to age, education, and marital and employment status.

As having a mental disorder, being an adolescent or older adult, having lower educational attainment, being unmarried, and being unemployed are identified as crossnational risk factors for suicide (Nock et al., 
TABLE 3

Logistic Regression Models for Suicide Attempt Versus No Attempt in Full Sample and Across Classes

\begin{tabular}{|c|c|c|c|c|}
\hline & Estimate & $S E$ & Odds ratio & $p$ \\
\hline \multicolumn{5}{|c|}{ Full sample $(N=1,321)$} \\
\hline TB & 0.25 & 0.14 & $1.29[0.96,1.73]$ & .08 \\
\hline $\mathrm{PB}$ & 1.01 & 0.12 & $2.74[2.14,3.50]$ & $<.01 * * *$ \\
\hline $\mathrm{CS}$ & 0.41 & 0.13 & $1.51[1.16,1.97]$ & $<.01 * * *$ \\
\hline $\mathrm{TB} \times \mathrm{PB}$ & 0.00 & 0.10 & $1.00[0.82,1.22]$ & .94 \\
\hline $\mathrm{CS} \times \mathrm{TB}$ & -0.03 & 0.14 & $0.97[0.73,1.27]$ & .83 \\
\hline $\mathrm{CS} \times \mathrm{PB}$ & -0.07 & 0.11 & $0.93[0.74,1.17]$ & .54 \\
\hline $\mathrm{TB} \times \mathrm{PB} \times \mathrm{CS}$ & -0.05 & 0.08 & $0.95[0.79,1.12]$ & .55 \\
\hline \multicolumn{5}{|c|}{ (X) No suicide ideation $(n=777)$} \\
\hline TB & 0.37 & 0.30 & $1.45[0.80,2.62]$ & .21 \\
\hline $\mathrm{PB}$ & 1.04 & 0.29 & $2.84[1.59,5.07]$ & $<.01 * * *$ \\
\hline CS & 0.74 & 0.27 & $2.10[1.23,3.59]$ & $<.01 * * *$ \\
\hline $\mathrm{TB} \times \mathrm{PB}$ & -0.04 & 0.28 & $0.95[0.54,1.65]$ & .86 \\
\hline $\mathrm{CS} \times \mathrm{TB}$ & 0.18 & 0.30 & $1.20[0.66,2.19]$ & .54 \\
\hline $\mathrm{CS} \times \mathrm{PB}$ & -0.11 & 0.26 & $0.89[0.53,1.49]$ & .66 \\
\hline $\mathrm{TB} \times \mathrm{PB} \times \mathrm{CS}$ & -0.55 & 0.25 & $0.57[0.35,0.94]$ & $.02 * *$ \\
\hline \multicolumn{5}{|c|}{ (A) Young, slight symptoms $(n=150)$} \\
\hline TB & -0.11 & 0.38 & $0.88[0.41,1.89]$ & .75 \\
\hline $\mathrm{PB}$ & -0.08 & 0.38 & $0.91[0.43,1.94]$ & .82 \\
\hline CS & 0.46 & 0.30 & $1.58[0.88,2.86]$ & .12 \\
\hline $\mathrm{TB} \times \mathrm{PB}$ & 0.65 & 0.33 & $1.91[0.99,3.70]$ & .05 \\
\hline $\mathrm{CS} \times \mathrm{TB}$ & -0.22 & 0.34 & $0.80[0.40,1.58]$ & .52 \\
\hline $\mathrm{CS} \times \mathrm{PB}$ & -0.21 & 0.32 & $0.80[0.42,1.51]$ & .49 \\
\hline $\mathrm{TB} \times \mathrm{PB} \times \mathrm{CS}$ & 0.19 & 0.30 & $1.21[0.67,2.19]$ & .51 \\
\hline \multicolumn{5}{|c|}{ (B) Older, slight symptoms $(n=188)$} \\
\hline TB & 0.17 & 0.36 & $1.18[0.58,2.42]$ & 64 \\
\hline $\mathrm{PB}$ & 0.47 & 0.40 & $1.60[0.72,3.54]$ & .24 \\
\hline CS & 0.47 & 0.36 & $1.60[0.79,3.25]$ & .18 \\
\hline $\mathrm{TB} \times \mathrm{PB}$ & -0.00 & 0.50 & $0.99[0.37,2.64]$ & .98 \\
\hline $\mathrm{CS} \times \mathrm{TB}$ & -0.51 & 0.41 & $0.59[0.26,1.33]$ & 21 \\
\hline $\mathrm{CS} \times \mathrm{PB}$ & 0.07 & 0.55 & $1.07[0.36,3.18]$ & .89 \\
\hline $\mathrm{TB} \times \mathrm{PB} \times \mathrm{CS}$ & 0.06 & 0.43 & $1.06[0.44,2.51]$ & .89 \\
\hline \multicolumn{5}{|c|}{ (C) Young, high symptoms $(n=124)$} \\
\hline ТВ & -0.56 & 0.54 & $0.56[0.19,1.66]$ & .30 \\
\hline $\mathrm{PB}$ & 0.77 & 0.35 & $2.16[1.07,4.35]$ & $.03 *$ \\
\hline CS & -0.33 & 0.50 & $0.71[0.26,1.92]$ & .50 \\
\hline $\mathrm{TB} \times \mathrm{PB}$ & 0.21 & 0.28 & $1.23[0.70,2.17]$ & .45 \\
\hline $\mathrm{CS} \times \mathrm{TB}$ & 0.68 & 0.51 & $1.98[0.71,5.47]$ & 18 \\
\hline $\mathrm{CS} \times \mathrm{PB}$ & 0.31 & 0.37 & $1.37[0.66,2.85]$ & .39 \\
\hline $\mathrm{TB} \times \mathrm{PB} \times \mathrm{CS}$ & -0.31 & 0.26 & $0.72[0.42,1.23]$ & .23 \\
\hline \multicolumn{5}{|c|}{ (D) Older, high symptoms $(n=82)$} \\
\hline TB & 0.45 & 0.65 & $1.58[0.44,5.67]$ & 48 \\
\hline $\mathrm{PB}$ & 0.34 & 0.59 & $1.41[0.43,4.55]$ & .56 \\
\hline CS & 0.02 & 0.89 & $1.02[0.17,5.90]$ & .98 \\
\hline $\mathrm{TB} \times \mathrm{PB}$ & 0.30 & 0.49 & $1.36[0.51,3.62]$ & .53 \\
\hline $\mathrm{CS} \times \mathrm{TB}$ & 0.58 & 0.75 & $1.79[0.40,7.88]$ & 43 \\
\hline $\mathrm{CS} \times \mathrm{PB}$ & 0.39 & 0.72 & $1.48[0.35,6.18]$ & .58 \\
\hline $\mathrm{TB} \times \mathrm{PB} \times \mathrm{CS}$ & -0.64 & 0.59 & $0.52[0.16,1.67]$ & .27 \\
\hline
\end{tabular}

Note. $\mathrm{TB}=$ thwarted belongingness, $\mathrm{PB}=$ perceived Burdensomeness, $\mathrm{CS}=$ capability for suicide, $x=$ interaction. ${ }^{*} p<.05, * * p<.025, * * * p<.01$. 


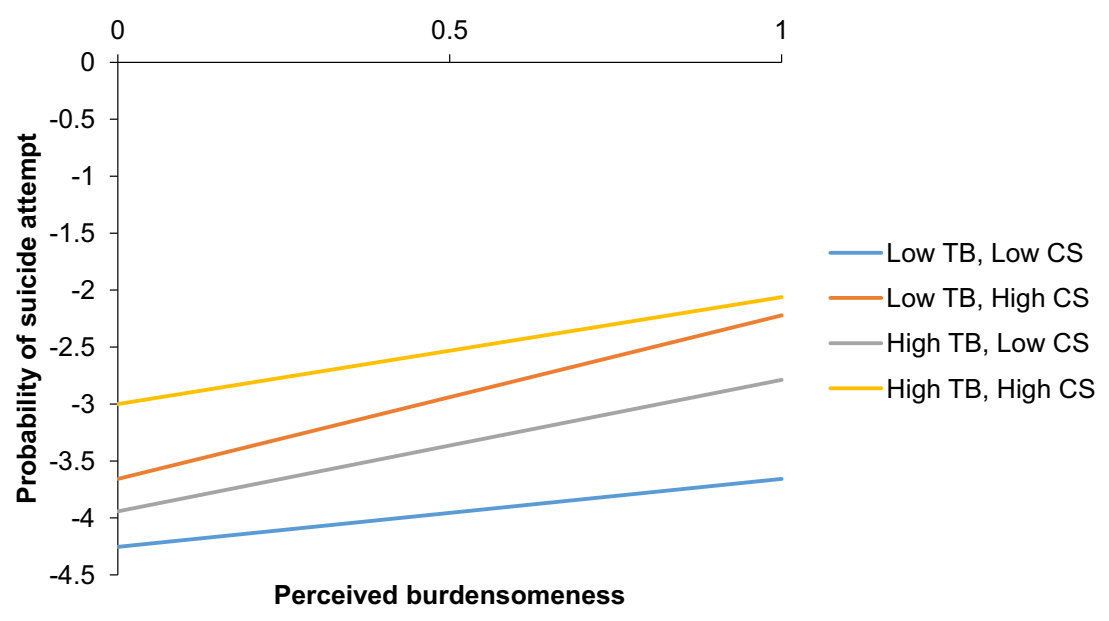

Figure 5. Interaction between thwarted belongingness (TB), perceived burdensomeness (PB), and capability for suicide $(\mathrm{CS})$ on suicide attempt in Class $\mathrm{X}$ (no suicide ideation). Low $=25$ th percentile, high $=75$ th percentile. TB, $\mathrm{PB}$, and $\mathrm{CS}$ scores are standardized.

2008), our findings provide further support for the role of mental health symptoms (even if only slightly elevated), age, education, and marital and employment status as risk factors associated with suicide ideation. Interestingly, the significant differences across the groups in relation to these sociodemographic variables were predominantly attributable to age, where younger groups tended to be single and currently studying, while older groups tended to be either mar$\mathrm{ried} /$ defacto or separated/divorced, and retired or not in the workforce. Although it is important to note that it is not necessarily the case that all samples with suicide ideation will fall into these groups, these profiles highlight the contextual nature of suicide risk and how it may manifest differently depending on life stage (e.g., loss of relationship through divorce or death experienced mainly in older groups), which has implications on the design and acceptance of targeted suicide prevention strategies and interventions.

In regard to our second aim, it was hypothesized that the highest levels of the interpersonal risk factors would be found in the groups reporting high levels of mental health symptoms. In line with this, results showed that high levels of $\mathrm{TB}$ and $\mathrm{PB}$ were found only in the four classes who reported suicide ideation in the past month, and not in the group without ideation. These findings support those of Dhingra et al. (2016), as well as the IPTS prediction that high levels of both TB and $\mathrm{PB}$ are risk factors for suicide ideation (Joiner, 2005). The results of the current study also indicated that the highest levels of TB and PB were found in the groups who experienced high levels of mental health symptoms. Here, groups significantly differed on levels of TB and PB based on severity of their mental health symptoms, and not age, supporting the relationship between mental health problems and suicide risk (Cavanagh, Carson, Sharpe, \& Lawrie, 2003; Nock, Hwang, Sampson, \& Kessler, 2010). Interestingly, a significant difference was not found in the levels of CS across any of the classes (including the no suicide ideation class). These findings suggest that CS may be quite stable over time and is perhaps less influenced by factors such as age, mental health symptom severity, and the absence or presence of suicide ideation. It also supports the theory's proposition of CS being less amenable to change in comparison with $\mathrm{TB}$ and $\mathrm{PB}$, where the latter may serve as more suitable targets for prevention and intervention. 
However, in interpreting these findings, it is important to note that recent investigations have raised concerns about the construct validity of the original 5-item measure used in this study (Ribeiro et al., 2014), limiting conclusions about the nature of capability for suicide.

Our third aim in this study, testing the predictions of the IPTS in each of the classes, was largely unsupported. The twoway interaction between $\mathrm{TB}$ and $\mathrm{PB}$ was significantly associated with suicide ideation experienced in the past month only in the young group with highly elevated mental health symptom scores. Here, TB did not seem to contribute to levels of suicide ideation significantly until individuals scored highly on $\mathrm{PB}$ (50th and 75th percentiles), suggesting that $\mathrm{PB}$ may play a greater role in contributing to suicide ideation (Figure 4). In addition to this, the model for the young group with highly elevated symptoms explained the most variance in suicide ideation compared to the other classes (approximately double), suggesting that the theory may be more strongly associated with suicide ideation in this demographic. This finding coincides with where a predominant amount of research on the IPTS has been focused (Ma et al., 2016).

In relation to the IPTS predictions regarding suicide attempt, the three-way interaction was significantly associated with attempt in the past year only for the group without suicide ideation. This model also explained the most variance, which was a surprising finding especially when compared to the full sample model, where only $\mathrm{PB}$ and CS were significant variables. One explanation for this may be related to the differences in time periods used for the suicide outcome measures, as the suicide ideation outcome focused on past month, while the suicide attempt outcome assessed attempts in the past year. Here, 23 (3\%) of the 777 individuals who reported no suicide ideation in the past month reported a suicide attempt in the past year. This finding indicates that although individuals without suicide ideation may have recovered in terms of their suicidal thoughts and behaviors, risk for suicide attempt may persist in the form of elevated interpersonal risk factors. Further investigation of the small subset of individuals with elevated interpersonal risk factors but no current suicide ideation may identify protective factors that mitigate feelings of isolation and burden. A further explanation for this finding may be that the interpersonal risk factors may be more intrinsic and stable in nature than previously thought. Such stability in these factors may have implications for the way we approach suicide prevention and strategies for intervention, as they may not be readily amenable to intervention. If this is the case, then other interpersonal targets, such as those underlying $\mathrm{TB}$ and $\mathrm{PB}$, may need to be identified that are more responsive to therapeutic intervention. Future research would benefit from examining the extent to which these interpersonal risk factors are amenable to change, as well as longitudinally examining their role and strength in contributing to vulnerability to suicide across the life span.

\section{Strengths and Limitations}

Strengths of this study include the large community-based sample, use of wellestablished and validated mental health measures, and novel application of LCA in relation to the IPTS. To our knowledge, this is the first study to test the IPTS predictions across subgroups derived by latent class analysis in a population-based sample. However, the study also had several limitations. First, because the study was cross-sectional, it is not possible to draw conclusions about the direction of the relationships. Second, although the sample was community based, it may not have been representative of the population as individuals with higher rates of psychopathology were recruited into the study. Third, measures used for the mental health constructs were brief self-report epidemiological scales as opposed to structured clinical assessments, and the differing time frames used 
to assess current specific disorders may have impacted temporal relationships with suicide outcomes. Fourth, the ACSS displayed low levels of internal consistency and may not be fully representative of the construct, limiting conclusions made about capability for suicide. Fifth, power to detect three-way interaction effects in the subgroups may have been limited due to smaller sample size. However, a three-way interaction effect was also not detected in the full sample, suggesting that samples in the thousands may be needed to observe the negligible magnitude of the three-way effects. Sixth, a downside of using LCA was that each of the subgroups had a restricted range of characteristics, reducing generalizability of the findings.

\section{REFERENCES}

Bastien, C. H., Vallières, A., \& Morin, C. M. (2001). Validation of the Insomnia Severity Index as an outcome measure for insomnia research. Sleep Medicine, 2, 297-307. https://doi. org/10.1016/S1389-9457(00)00065-4

Batterham, P. J. (2014). Recruitment of mental health survey participants using internet advertising: Content, characteristics and cost effectiveness. International fournal of Methods in Psychiatric Research, 23, 184-191. https://doi.org/ 10.1002/mpr.1421

Batterham, P. J., Calear, A. L., \& Christensen, H. (2013). Correlates of suicide stigma and suicide literacy in the community. Suicide and Life-Threatening Behavior, 43, 406417. https://doi.org/10.1111/sltb.2013.43.issue-4

Batterham, P. J., Calear, A. L., \& SpiJKER, B. A. J. (2015). The specificity of the interpersonal-psychological theory of suicidal behavior for identifying suicidal ideation in an online sample. Suicide and Life-Threatening Behavior, 45, 448460. https://doi.org/10.1111/sltb.12140

Batterham, P. J., Mackinnon, A. J., \& Christensen, H. (2016). Community-based validation of the Social Phobia Screener (SOPHS). Assessment, 24, 1-12. https://doi.org/10.1177/ 1073191116636448

Bryan, C. J., Clemans, T. A., \& HernanDEZ, A. M. (2012). Perceived burdensomeness, fearlessness of death, and suicidality among deployed military personnel. Personality and Individual Differences, 52, 374-379. https://doi.org/ 10.1016/j.paid.2011.10.045

\section{CONCLUSIONS}

The present study highlighted the utility of LCA in testing the predictions of the IPTS in an online community survey of Australian adults, contributing a number of useful insights into the nature of the interpersonal factors in the community. Support was provided for the relationship between severity of mental health symptoms and higher levels of thwarted belongingness and perceived burdensomeness in people with suicide ideation. Lack of support for the IPTS predictions regarding suicide ideation and attempt outcomes across the subgroups and full sample in this study raises some questions around the broad applicability of the theory.

Bush, K., Kivlahan, D. R., McDonell, M. B., Finn, S. D., \& Bradley, K. A. (1998). The AUDIT Alcohol Consumption Questions (AUDIT-C): An effective brief screening test for problem drinking. Archives of Internal Medicine, 158, 1789-1795. https://doi.org/10.1001/arch inte.158.16.1789

Cavanagh, J. T., Carson, A. J., Sharpe, M., \& Lawrie, S. M. (2003). Psychological autopsy studies of suicide: A systematic review. Psychological Medicine, 33, 395-405. https://doi. org/10.1017/S0033291702006943

Christensen, H., Batterham, P. J., Mackinnon, A. J., Donker, T., \& Soubelet, A. (2014). Predictors of the risk factors for suicide identified by the interpersonal-psychological theory of suicidal behaviour. Psychiatry Research, 219, 290-297. https://doi.org/10.1016/j.psychres. 2014.05.029

Cukrowicz, K. C., Jahn, D. R., Graham, R. D., Poindexter, E. K., \& Williams, R. B. (2013). Suicide risk in older adults: Evaluating models of risk and predicting excess zeros in a primary care sample. Fournal of Abnormal Psychology, 122, 1021-1030. https://doi.org/10.1037/ a0034953

Czyz, E. K., Berona, J., \& King, C. A. (2015). A prospective examination of the interpersonal-psychological theory of suicidal behavior among psychiatric adolescent inpatients. Suicide and Life-Threatening Behavior, 45, 243-259. https://doi.org/10.1111/sltb.2015.45.is sue-2 
Dhingra, K., Boduszek, D., \& Klonsky, D. (2016). Empirically derived subgroups of selfinjurious thoughts and behavior: Application of latent class analysis. Suicide and Life-Threatening Behavior, 46, 1-14. https://doi.org/10.1111/sltb. 12232

Fassaert, T., De Wit, M. A., TuinebreiJer, W. C., Wouters, H., Verhoeff, A. P., Beekman, A. T., ET AL. (2009). Psychometric properties of an interviewer-administered version of the Kessler Psychological Distress scale (K10) among Dutch, Moroccan and Turkish respondents. International 7ournal of Methods in Psychiatric Research, 18, 159-168. https://doi.org/10. 1002/mpr.v18:3

Franklin, J. C., Ribeiro, J. D., Fox, K. R., Bentley, K. H., Kleiman, E. M., Huang, X., ET AL. (2016). Risk factors for suicidal thoughts and behaviors: A meta-analysis of 50 years of research. Psychological Bulletin, 143, 187-232. https://doi.org/10.1037/bul0000084

Freedenthal, S., Lamis, D. A., Osman, A., Kahlo, D., \& Gutierrez, P. M. (2014). Evaluation of the psychometric properties of the interpersonal needs questionannire-12 in samples of men and women. Fournal of Clinical Psychology, 67, 609-623. https://doi.org/10.1002/jclp.20782

GunN, J. F., \& Lester, D. (2014). Theories of suicide: Past, present and future. Springfield, IL: Charles C Thomas.

JoIner, T. E., JR. (2005). Why people die by suicide. Cambridge, MA: Harvard University Press.

Kessler, R. C., Adler, L., Ames, M., Demler, O., Faraone, S., Hiripi, E., et al. (2005). The world health organization adult ADHD Self-Report Scale (ASRS): A short screening scale for use in the general population. Psychological Medicine, 35, 245-256. https://doi. org/10.1017/S0033291704002892

Kessler, R. C., Andrews, G., Colpe, L. J., Hiripi, E., Mroczek, D. K., Normand, S.-L. T., ET AL. (2002). Short screening scales to monitor population prevalences and trends in nonspecific psychological distress. Psychological Medicine, 32, 959-976. https://doi.org/10.1017/ S0033291702006074

KIM, S., \& YANG, E. (2015). Suicidal ideation in gay men and lesbians in South Korea: A test of the interpersonal-psychological model. Suicide and Life-Threatening Behavior, 45, 98-110. https://doi.org/10.1111/sltb.2015.45.issue-1

Kleiman, E. M., Law, K. C., \& Anestis, M. D. (2014). Do theories of suicide play well together? Integrating components of the hopelessness and interpersonal psychological theories of suicide. Comprebensive Psychiatry, 55, 431-438. https://doi.org/10.1016/j.comppsych.2013.10.015

Kroenke, K., Spitzer, R. L., \& WiLLIAMS, J. B. (2001). The PHQ-9: Validity of a brief depression severity measure. Fournal of General Internal Medicine, 16, 606-613. https://doi. org/10.1046/j.1525-1497.2001.016009606.x

Lazarsfeld, P. F., \& Henry, N. W. (1968). Latent structure analysis. Boston, MA: Houghton Mifflin.

Logan, J., Hall, J., \& Karch, D. (2011). Suicide categories by patterns of known risk factors. Archives of General Psychiatry, 68, 935-941. https://doi.org/10.1001/archgenpsychiatry.2011.85 LÖWE, B., GräFe, K., ZipfEl, S., SPITZer, R. L., Herrmann-Lingen, C., Witte, S., et al. (2003). Detecting panic disorder in medical and psychosomatic outpatients: Comparative validation of the Hospital Anxiety and Depression Scale, the Patient Health Questionnaire, a screening question, and physicians' diagnosis. Journal of Psychosomatic Research, 55, 515-519. https://doi.org/10.1016/S0022-3999(03)00072-2

Ma, J., Batterham, P. J., Calear, A. L., \& Han, J. (2016). A systematic review of the predictions of the interpersonal-psychological theory of suicidal behaviour. Clinical Psychology Review, 46, 34-45. https://doi.org/10.1016/j.cpr. 2016.04.008

McLachlan, G., \& Peel, D. (2000). Finite mixture models. New York, NY: Wiley. https://doi.org/10.1002/0471721182

MuthéN, L. K., \& MuthéN, B. O. (1998-2010). Mplus user's guide (6th ed.). Los Angeles, CA: Muthén \& Muthén.

Nock, M. K., Borges, G., Bromet, E. J., Cha, C. B., Kessler, R. C., \& Lee, S. (2008). Suicide and suicidal behavior. Epidemiologic Reviews, 30, 133-154. https://doi.org/10.1093/ epirev/mxn002

Nock, M. K., Hwang, I., Sampson, N. A., \& Kessler, R. C. (2010). Mental disorders, comorbidity and suicidal behavior: Results from the National Comorbidity Survey Replication. Molecular Psychiatry, 15, 868-876. https://doi.org/ 10.1038/mp.2009.29

Nylund, K. L., Asparouhov, T., \& Muthén, B. O. (2007). Deciding on the number of classes in latent class analysis and growth mixture modeling: A Monte Carlo simulation study. Structural Equation Modeling, 14, 535-569. https:// doi.org/10.1080/10705510701575396

O'Keefe, V. M., Wingate, L. R., Tucker, R. P., Rhoades-Kerswill, S., Slish, M. L., \& Davidson, C. L. (2014). Interpersonal suicide risk for American Indians: Investigating thwarted belongingness and perceived burdensomeness. Cultural Diversity and Ethnic Minority Psychology, 20, 61-67. https://doi.org/10.1037/ a0033540

Patel, V., Araya, R., Chowdhary, N., King, M., Kirkwood, B., Nayak, S., ET AL. (2008). Detecting common mental disorders in 
primary care in India: A comparison of five screening questionnaires. Psychological Medicine, $38,221-228$.

Posner, K., Brown, G. K., Stanley, B., Brent, D. A., Yershova, K. V., Oquendo, M. A., ET AL. (2011). The Columbia-Suicide Severity Rating Scale: Initial validity and internal consistency findings from three multisite studies with adolescents and adults. American fournal of Psychiatry, 168, 1266-1277. https://doi.org/10. 1176/appi.ajp.2011.10111704

Ribeiro, J. D., Witte, T. K., Van Orden, K. A., Selby, E. A., Gordon, K. H., Bender, T. W., ET AL. (2014). Fearlessness about death: The psychometric properties and construct validity of the revision to the Acquired Capability for Suicide Scale. Psychological Assessment, 26, 115-126. https://doi.org/10.1037/a0034858

Rueter, M. A., Holm, K. E., McGeorge, C. R., \& Conger, R. D. (2008). Adolescent suicidal ideation subgroups and their association with suicidal plans and attempts in young adulthood. Suicide and Life-Threatening Behavior, 38 , 564-575. https://doi.org/10.1521/suli.2008.38.5. 564

Smith, A. R., Ribeiro, J. D., MikolajeWSki, A., TaYlor, J., Joiner, T. E., \& Iacono, W. G. (2012). An examination of environmental and genetic contributions to the determinants of suicidal behavior among male twins. Psychiatry Research, 197, 60-65. https://doi.org/10.1016/j. psychres.2012.01.010

Smith, P. N., Wolford-Clevenger, C., Selwyn, C. N., Poindexter, E., Lechner, W., Grant, D. M., ET AL. (2015). An exploratory analysis of the relations between the rate of physiological habituation, the acquired capability for suicide, and acute risk factors for suicide. Journal of Aggression, Conflict and Peace Research, 7, 139-148. https://doi.org/10.1108/JACPR-072014-0130

Spitzer, R., Kroenke, K., \& Williams, J. (1999). Validation and utility of a self-report Version of PRIME-MD: The PHQ Primary Care Study. Fournal of the American Medical Association, 282, 1737-1744. https://doi.org/10. 1001/jama.282.18.1737

Spitzer, R. L., Kroenke, K., Williams, J. B. W., \& LöwE, B. (2006). A brief measure for assessing generalized anxiety disorder: The GAD7. Archives of Internal Medicine, 166, 1092-1097. https://doi.org/10.1001/archinte.166.10.1092

StataCorp (2015). Stata statistical software: Release 14. College Station, TX: StataCorp LP.
Van Orden, K. A., Cukrowicz, K. C., WitTe, T. K., \& Joiner, T. E. (2012). Thwarted belongingness and perceived burdensomeness: Construct validity and psychometric properties of the Interpersonal Needs Questionnaire. Psychological Assessment, 24, 197-215. https://doi.org/ 10.1037/a0025358

Van Orden, K. A., Witte, T. K., Cukrowicz, K. C., Braithwaite, S. R., Selby, E. A., \& JoIner, T. E. (2010). The interpersonal theory of suicide. Psychological Review, 117, 575600. https://doi.org/10.1037/a0018697

Van Orden, K. A., Witte, T. K., GorDON, K. H., Bender, T. W., \& JoIner, T. E., JR. (2008). Suicidal desire and the capability for suicide: Tests of the interpersonal-psychological theory of suicidal behavior among adults. Fournal of Consulting and Clinical Psychology, 76, 72-83. https://doi.org/10.1037/0022-006X.76.1.72

Van Spijker, B. A. J., Batterham, P. J., Calear, A. L., Farrer, L., Christensen, H. Reynolds, J., ET AL. (2014). The Suicidal Ideation Attributes Scale (SIDAS): Community-based validation study of a new scale for the measurement of suicidal ideation. Suicide and Life-Threatening Behavior, 44, 408-419. https://doi.org/10. 1111/sltb.2014.44.issue-4

World Health Organization (2014). Preventing suicide: A global imperative (pp. 1-89). Geneva, Switzerland: Author.

WITTKAMPF, K. A., BAas, K. D., VAN Weert, H. C., Lucassen, P., \& Schene, A. H. (2011). The psychometric properties of the panic disorder module of the Patient Health Questionnaire (PHQ-PD) in high-risk groups in primary care. Fournal of Affective Disorders, 130, 260-267. https://doi.org/10.1016/j.jad.2010.10.030

Wong, Y., Koo, K., Tran, K. K., Chiu, Y.-C., \& Mok, Y. (2011). Asian American college students' suicide ideation: A mixed-methods study. Fournal of Counseling Psychology, 58, $197-$ 209. https://doi.org/10.1037/a0023040

Wong, J. Y., \& Maffini, C. S. (2011). Predictors of asian american adolescents' suicide attempts: A latent class regression analysis. Fournal of Youth and Adolescence, 40, 1453-1464. https://doi.org/10.1007/s10964-011-9701-3

Manuscript Received: March 29, 2017

Revision Accepted: August 24, 2017 